\title{
Error Estimates for Spatially Discrete Approximations of Semilinear Parabolic Equations with Initial Data of Low Regularity
}

\author{
By M. Crouzeix, V. Thomée, and L. B. Wahlbin
}

\begin{abstract}
Semidiscrete finite element methods for a semilinear parabolic equation in $R^{d}, d \leq 3$, were considered by Johnson, Larsson, Thomee, and Wahlbin. With $h$ the discretization parameter, it was proved that, for compatible and bounded initial data in $H^{\alpha}$, the convergence rate is essentially $O\left(h^{2+\alpha}\right)$ for $t$ positive, and for $\alpha=0$ this was seen to be best possible. Here we shall show that for $0 \leq \alpha<2$ the convergence rate is, in fact, essentially $O\left(h^{2+2 \alpha}\right)$, which is sharp.
\end{abstract}

0. Introduction. The aim of this paper is to improve certain results from Johnson, Larsson, Thomée, and Wahlbin [2]. In this introduction we shall describe these results and motivate and state our new findings.

The investigations in [2] are concerned with nonsmooth data error estimates for spatially discrete approximations to the solution of the initial-boundary value problem

$$
\begin{array}{ll}
u_{t}-\Delta u=f(u) & \text { in } \Omega \times I, \quad I=\left(0, t^{*}\right], \\
u=0 & \text { on } \partial \Omega \times I, \\
u(0)=v & \text { in } \Omega,
\end{array}
$$

where $\Omega$ is a bounded domain in $R^{d}, d=1,2$, or 3 , with smooth boundary $\partial \Omega$, and $f$ is a smooth function on $R$ which is bounded together with an appropriate number of its derivatives. (For a discussion of this assumption, see [2, Section 3].) It is assumed throughout that $v$, and hence $u$, is bounded.

The spatially discrete approximation $u_{h}(t)$ is sought in a finite-dimensional space $S_{h} \subset H_{0}^{1}(\Omega)$ and is defined by

$$
\begin{aligned}
& \left(u_{h, t}, \chi\right)+\left(\nabla u_{h}, \nabla \chi\right)=\left(f\left(u_{h}\right), \chi\right) \text { for } \chi \in S_{h}, t \in I, \\
& u_{h}(0)=P_{0} v,
\end{aligned}
$$

where $(v, w)$ is the standard inner product in $L_{2}=L_{2}(\Omega)$, and $P_{0}$ is the orthogonal projection in $L_{2}$ onto $S_{h}$. It is assumed that the family $\left\{S_{h}\right\}$ is such that the elliptic projection $P_{1}$, the orthogonal projection onto $S_{h}$ with respect to the Dirichlet inner product $(\nabla v, \nabla w)$, has an error of order $h^{r}, r \geq 2$ integer, or, more precisely,

$$
\left\|P_{1} w-w\right\| \leq C h^{r}\|w\|_{r} \text { for } w \in H^{r} \cap H_{0}^{1},
$$

where $\|\cdot\|$ and $\|\cdot\|_{r}$ denote the standard norms in $L_{2}$ and $H^{r}=H^{r}(\Omega)$, respectively.

It was first proved that (cf. also Helfrich [1])

$$
\left\|u_{h}(t)-u(t)\right\| \leq C(R) h^{2} \log (1 / h) t^{-1} \text { for }\|v\| \leq R, t \in I .
$$

Received April 20, 1988.

1980 Mathematics Subject Classification (1985 Revision). Primary 65M20, 65M60. 
It was then shown that, in contrast to the linear case, this result is essentially sharp, in the sense that an estimate of the form

$$
\left\|u_{h}\left(t_{0}\right)-u\left(t_{0}\right)\right\| \leq C\left(R, t_{0}\right) h^{\sigma} \text { for }\|v\|_{L_{\infty}} \leq R,
$$

with $\|v\|_{L_{\infty}}$ the norm in $L_{\infty}=L_{\infty}(\Omega)$, cannot hold for any $\sigma>2$ and $t_{0}>0$, regardless of the order of approximation of the family $\left\{S_{h}\right\}$. This was first made plausible by considering the following system with $2 \pi$-periodic boundary conditions:

$$
\begin{array}{ll}
u_{1, t}=u_{1, x x}+f\left(u_{2}\right) & \text { where } f(y)=4 y^{2} \text { for }|y| \leq 1, \\
u_{2, t}=u_{2, x x} & \text { on }[-\pi, \pi] \times(0, \infty), \\
u_{1}(0)=0, \quad u_{2}(0)=v_{2} . &
\end{array}
$$

For the approximate solution, let $h=1 / n$, with $n$ a positive integer, and set

$$
S_{h}=\operatorname{span}\{1, \cos x, \sin x, \ldots, \cos (n-1) x, \sin (n-1) x\},
$$

which has order of approximation $O\left(h^{r}\right)$ for any positive $r$. With $v_{2}(x)=\cos n x$, one has $P_{0} v \equiv 0$, and the Galerkin solution vanishes identically. Hence the error equals the exact solution, which is easily checked to be

$$
u=\left(u_{1}, u_{2}\right)=\left(n^{-2}\left(1-e^{-2 n^{2} t}\right)\left(1+e^{-2 n^{2} t} \cos 2 n x\right), e^{-n^{2} t} \cos n x\right),
$$

whence, for large $n$,

$$
\left\|u_{h}\left(t_{0}\right)-u\left(t_{0}\right)\right\|=\left\|u\left(t_{0}\right)\right\| \simeq C n^{-2}=C h^{2} .
$$

Since $v$ is bounded independently of $n$, this contradicts $(0.3)$ for $\sigma>2$.

Note that it is natural to use $f(y) \simeq y^{2}$ as a model smooth nonlinearity, since the linear part of a Taylor expansion of $f$ can be combined with $u_{x x}$ to form a linear elliptic operator.

To analyze the case of slightly more regular data, a subset $\mathscr{F}_{\alpha}$ of $H^{\alpha}$ was introduced in [2], together with a defining functional $F_{\alpha}(v)$. The set $\mathscr{F}_{\alpha}$ may be thought of as consisting of those $v \in H^{\alpha} \cap L_{\infty}$ for which sufficient compatibility with the differential equation holds at $\partial \Omega$ at $t=0$ for $\|u(t)\|_{\alpha}$ to be bounded even as $t$ approaches 0 . Here, $H^{\alpha}$ is defined for noninteger $\alpha$ by interpolation. It was shown that if $v \in \mathscr{F}_{\alpha}$, then, for any $\sigma$ with $0 \leq \sigma<2$ and such that $\sigma+\alpha \leq r$,

$$
\left\|u_{h}(t)-u(t)\right\| \leq C(R) h^{\sigma+\alpha} t^{-\sigma / 2} \text { for } F_{\alpha}(v) \leq R, t \in I .
$$

The order of convergence was thus shown to be essentially two orders higher than the initial regularity.

In order to try to modify the above counterexample, to see whether this latter estimate is sharp, we now choose $v_{2, \alpha}(x)=n^{-\alpha} \cos n x$. Interpreting the $H^{\alpha}$ norm for a periodic function $v=\sum_{j=-\infty}^{\infty} c_{j} e^{i j x}$ to be

$$
\|v\|_{\alpha, \#}=\left(\sum_{j=-\infty}^{\infty} j^{2 \alpha}\left|c_{j}\right|^{2}\right)^{1 / 2}
$$

we have $\left\|v_{2, \alpha}\right\|_{\alpha, \#}=$ constant, independent of $n$. Now the solution of (0.4) is $u=\left(u_{1, \alpha}, u_{2, \alpha}\right)=\left(n^{-2 \alpha} u_{1}(x, t), n^{-\alpha} u_{2}(x, t)\right)$, where $\left(u_{1}, u_{2}\right)$ are given by $(0.5)$, and, by the same reasoning as above,

$$
\left\|u_{h}\left(t_{0}\right)-u\left(t_{0}\right)\right\|=\left\|u\left(t_{0}\right)\right\| \simeq C n^{-2-2 \alpha}=C h^{2+2 \alpha} \text { for large } n .
$$


This therefore does not show the $O\left(h^{2+\alpha}\right)$ error estimate of [2] to be sharp.

In this paper we shall show that the order of convergence suggested by the modified counterexample is the correct one. Under an additional approximation assumption for the elliptic projection (see (1.3)), we shall prove that if $0 \leq \alpha<2$, $2+2 \alpha \leq r$, then we have, with $\nu=\nu(\alpha)<1$,

$$
\begin{array}{r}
\left\|u_{h}(t)-u(t)\right\| \leq C(R) h^{2+2 \alpha}\left(t^{-(2+\alpha) / 2}+t^{-\nu} \log (1 / h)\right) \\
\text { for } F_{\alpha}(v) \leq R, t \in I,
\end{array}
$$

which is an improvement over (0.6) by a factor $h^{\alpha}$ in the case considered. In the present range of $\alpha, F_{\alpha}(v)$ is equivalent to $\max \left(\|v\|_{\alpha},\|v\|_{L_{\infty}}\right)$.

We remark that also the scalar spline counterexample to $(0.3)$ given in [2, Section 6] can easily be modified to show that no better rate of convergence than $O\left(h^{2+2 \alpha}\right)$ is possible in a result such as (0.7).

The restriction $\alpha<2$ in (0.7) is probably due to our techniques of proof; our result covers optimal-order estimates for finite element spaces up to isoparametric quintics.

The proof of the result (0.7) will be given in Section 2 below. It requires some refined error estimates for a linear nonhomogeneous parabolic equation, which we shall present in Section 1.

In estimates like (0.7) it is natural to ask if the logarithmic factor can be removed, and it is a popular pastime to attempt to do so. We have not been able to accomplish this in general, but shall indicate at the end of Section 2 how this can be done in the cases $0 \leq \alpha \leq 1, r \geq 4$ and $\alpha=0, r \geq 3$.

In [2] an $O\left(h^{\sigma+\alpha}\right)$ error estimate was obtained also in maximum norm for $v \in \mathscr{F}_{\alpha}$, $\sigma+\alpha \leq r$ and $t>0$, and a similar estimate was also demonstrated for the error in the gradient. These estimates were consequences of the $L_{2}$ norm error estimates (0.6) and may therefore be improved analogously to (0.7), as will be briefly indicated in Section 3.

1. Estimates for a Linear Nonhomogeneous Equation. In this section we shall consider the linear problem

$$
\begin{array}{ll}
u_{t}-\Delta u=g & \text { in } \Omega \times I, \\
u=0 & \text { on } \partial \Omega \times I, \\
u(0)=v & \text { in } \Omega,
\end{array}
$$

where $g$ is a function of $(x, t)$ which we assume to be in $L_{\infty}\left(L_{\infty}\right)=L_{\infty}\left(I ; L_{\infty}(\Omega)\right)$ (we shall frequently suppress the dependence of the spatial domain and the time interval in our notation when they equal $\Omega$ and $I$, respectively). The semidiscrete Galerkin method is to find $u_{h}: I \rightarrow S_{h}$ such that

$$
\begin{aligned}
& \left(u_{h, t}, \chi\right)+\left(\nabla u_{h}, \nabla \chi\right)=(g, \chi) \text { for } \chi \in S_{h}, t \in I, \\
& u_{h}(0)=P_{0} v .
\end{aligned}
$$

We shall first recall some known results for the case of the homogeneous equation, i.e., $g=0$ (see Thomée [4] and references therein). For this purpose we introduce the space $\dot{H}^{\alpha}=\dot{H}^{\alpha}(\Omega)$ defined for any real $\alpha$ by the norm $|v|_{\alpha}=\left\|(-\Delta)^{\alpha / 2} v\right\|$. For $\alpha$ a positive integer, $\dot{H}^{\alpha}(\Omega)=\left\{v \in H^{\alpha}(\Omega) ; \Delta^{j} v=0\right.$ on $\partial \Omega$ for $\left.j<\alpha / 2\right\}$ (see 
$[4$, p. 34]). From now on we make the assumption that the error in the elliptic projection satisfies

$$
\begin{aligned}
& \left|P_{1} w-w\right|_{-l} \leq C h^{\gamma}\|w\|_{\gamma-l} \\
& \quad \text { for } 0 \leq \gamma \leq r, \gamma-l \geq 1,0 \leq l \leq r-2, w \in H^{\gamma-l} \cap H_{0}^{1} .
\end{aligned}
$$

Note that the approximation order $\gamma$ never exceeds $r$. Thus, this assumption is satisfied, e.g., for isoparametric elements of degree $r-1$, if the mesh domains are kept inside $\Omega$. We also introduce the notation $E(t), E_{h}(t)$, and $F_{h}(t)=E_{h}(t) P_{0}-$ $E(t)$ for the solution operator of the initial value problem (1.1) with $g=0$, its discrete counterpart, and the resulting error. For initial data $v \in \dot{H}^{\alpha}$ we have the error estimate

$$
\left|F_{h}(t) v\right|_{-l} \leq C h^{\gamma} t^{-(\gamma-\alpha-l) / 2}|v|_{\alpha} \quad \text { for } t \in I, l+\alpha \leq \gamma \leq r .
$$

For $\alpha=0$, this follows from the corresponding estimate with $l=0$ (cf. [4, Chapter 3]) by a simple duality argument, since $F_{h}(t)$ is selfadjoint on $L_{2}$. We then obtain the general result for $(-l, \alpha)$ by interpolation between the results for $(0, \gamma)$ and $(-l \gamma /(\gamma-\alpha), 0)$. These estimates depend on a corresponding smoothness property of the exact solution, namely, with $D_{t}=\partial / \partial t$,

$$
\left|D_{t}^{s} E(t) v\right|_{\mu} \leq C t^{-(\mu-\alpha+2 s) / 2}|v|_{\alpha} \quad \text { for } t \in I, \mu+2 s \geq \alpha,
$$

and its discrete analogue.

Our purpose is now to generalize (1.4) to the nonhomogeneous equation in (1.1), with application to the semilinear equation (0.1) in mind. We shall make the temporary hypothesis that the exact solution has the appropriate behavior for small $t$, and then verify this at the end of the section for the case that $g(x, t)=f(u(x, t))$ where $u$ is the solution of $(0.1)$. Here and below we denote, for various functions $w$ on $I$,

$$
\tilde{w}(t)=\int_{0}^{t} w(s) d s .
$$

THEOREM 1.1. Let $\alpha \geq 0$ and $v \in \dot{H}^{\alpha}$ if $\alpha \leq 1$, and let $g$ be such that the solution $u$ of (1.1) satisfies

$$
\left\|D_{t}^{j} u(t)\right\|_{\mu} \leq C t^{-(\mu-\alpha+2 j) / 2} \quad \text { for } \alpha \leq \mu \leq 2+2 \alpha, j=0,1, t \in I,
$$

and

$$
\|\tilde{u}(t)\|_{\mu} \leq C t^{1-(\mu-\alpha) / 2} \quad \text { for } \alpha \leq \mu \leq 2+\alpha, t \in I .
$$

Then we have for the error in (1.2)

$$
\begin{aligned}
& \left|u_{h}(t)-u(t)\right|_{-l} \leq C h^{\gamma} t^{-(\gamma-\alpha-l) / 2} \\
& \qquad \text { for } \gamma \leq \min (2+2 \alpha, r), 0 \leq l \leq r-2, \gamma-\alpha-l \geq 0, t \in I .
\end{aligned}
$$

For the purpose of proof we introduce a discrete analogue $T_{h}: L_{2} \rightarrow S_{h}$ of $T=(-\Delta)^{-1}$, defined by

$$
\left(\nabla T_{h} f, \nabla \chi\right)=(f, \chi) \text { for } \chi \in S_{h},
$$

and then, as is easily seen, the error $e=u_{h}-u$ in (1.2) satisfies

$$
T_{h} e_{t}+e=\rho \text { for } t \in I,
$$


where $\rho=-\left(P_{1}-I\right) u$. We shall also use the discrete negative seminorm $|v|_{-j, h}=$ $\left(T_{h}^{j} v, v\right)^{1 / 2}$. We recall (cf. $[4$, p. 82]) that for $0 \leq j \leq r-2$ this seminorm is related to the norm in $\dot{H}^{-j}$ by

$$
|w|_{-j, h} \leq C\left(|w|_{-j}+h^{j}\|w\|\right)
$$

and

$$
|w|_{-j} \leq C\left(|w|_{-j, h}+h^{j}\|w\|\right) .
$$

We begin with the following lemma.

LEMMA 1.1. Let $e$ be a solution of (1.9) with $T_{h} e(0)=0$. For any positive integer $k$, there exists $C=C(k)$ such that for any nonnegative integer $j$,

$$
t^{k}|e(t)|_{-j, h} \leq C \sup _{s \leq t}\left\{s^{k+1}\left|\rho_{t}(s)\right|_{-j, h}+s^{k}|\rho(s)|_{-j, h}+s^{k-1 / 2}|e(s)|_{-j-1, h}\right\}
$$

for $t \in I$.

Proof. Set temporarily $\langle v, w\rangle=\left(T_{h}^{j} v, w\right),|v|=\langle v, v\rangle^{1 / 2}=|v|_{-j, h}$ and $|v|_{*}=$ $|v|_{-j-1, h}=\left\langle T_{h} v, v\right\rangle^{1 / 2}$. Note that $T_{h}$ is a positive semidefinite operator with respect to the semi-inner product $\langle v, w\rangle$.

Taking the inner product of (1.9) against $e_{t}$ we have

$$
\frac{1}{2} \frac{d}{d t}|e|^{2} \leq\left\langle\rho, e_{t}\right\rangle=\frac{d}{d t}\langle\rho, e\rangle-\left\langle\rho_{t}, e\right\rangle
$$

and, multiplying this inequality by $t^{2 k+1}$,

$$
\begin{aligned}
\frac{1}{2} \frac{d}{d t}\left(t^{2 k+1}|e|^{2}\right) \leq & \frac{d}{d t}\left(t^{2 k+1}\langle\rho, e\rangle\right)-t^{2 k+1}\left\langle\rho_{t}, e\right\rangle \\
& +\frac{1}{2}(2 k+1) t^{2 k}|e|^{2}-(2 k+1) t^{2 k}\langle\rho, e\rangle .
\end{aligned}
$$

Integrating, we obtain

$$
\begin{aligned}
t^{2 k+1}|e|^{2} \leq C\left\{t^{2 k+1}|\langle\rho, e\rangle|+\left|\int_{0}^{t} s^{2 k+1}\left\langle\rho_{t}, e\right\rangle d s\right|\right. & +\int_{0}^{t} s^{2 k}|e|^{2} d s \\
& \left.+\left|\int_{0}^{t} s^{2 k}\langle\rho, e\rangle d s\right|\right\} .
\end{aligned}
$$

Cauchy-Schwarz' inequality and a kickback argument then give

$$
\begin{aligned}
t^{2 k+1}|e(t)|^{2} \leq C\left\{t^{2 k+1}|\rho(t)|^{2}+\int_{0}^{t}\left(s^{2 k+2}\left|\rho_{t}\right|^{2}+\right.\right. & \left.s^{2 k}|\rho|^{2}\right) d s \\
& \left.+\int_{0}^{t} s^{2 k}|e|^{2} d s\right\} .
\end{aligned}
$$

We proceed to estimate the last term on the right. We take the inner product of (1.9) by $e$ to obtain

$$
\frac{1}{2} \frac{d}{d t}|e|_{*}^{2}+|e|^{2}=\langle\rho, e\rangle
$$

and multiply this by $t^{2 k}$, whence

$$
\frac{1}{2} \frac{d}{d t}\left(t^{2 k}|e|_{*}^{2}\right)+t^{2 k}|e|^{2}=t^{2 k}\langle\rho, e\rangle-k t^{2 k-1}|e|_{*}^{2} .
$$


Integrating and using Cauchy-Schwarz' inequality on the first term on the right, we find

$$
\int_{0}^{t} s^{2 k}|e|^{2} d s \leq C\left\{\int_{0}^{t} s^{2 k}|\rho|^{2} d s+\int_{0}^{t} s^{2 k-1}|e|_{*}^{2} d s\right\}
$$

Inserting this result into (1.13), we have

$$
t^{2 k+1}|e(t)|^{2} \leq C\left\{t^{2 k+1}|\rho(t)|^{2}+\int_{0}^{t}\left(s^{2 k+2}\left|\rho_{t}\right|^{2}+s^{2 k}|\rho|^{2}\right) d s+\int_{0}^{t} s^{2 k-1}|e|_{*}^{2} d s\right\}
$$

and the desired result (1.12) follows upon dividing by $t$. This proves Lemma 1.1.

We shall also need the following result from [4, p. 42].

LEMMA 1.2. There exists a constant $C$ such that for any nonnegative integer $j$,

$$
t|e(t)|_{-j, h} \leq C \sup _{s \leq t}\left\{s^{2}\left|\rho_{t}(s)\right|_{-j, h}+s|\rho(s)|_{-j, h}+|\tilde{\rho}(s)|_{-j, h}\right\} .
$$

We are now ready for the

Proof of Theorem 1.1. We shall prove the theorem successively for increasing values of the gap variable $\gamma-\alpha-l$ and start with $0 \leq \gamma-\alpha-l \leq 2$. Within this range we first consider $\gamma \leq l+1$. With the above notation, the solution of (1.1) may be written, by Duhamel's principle, as

$$
u(t)=E(t) v+\int_{0}^{t} E(t-s) g(s) d s
$$

and similarly for the solution of (1.2), so that by subtraction

$$
e(t)=F_{h}(t) v+\int_{0}^{t} F_{h}(t-s) g(s) d s
$$

Here, by (1.4),

$$
\left|F_{h}(t-s) g(s)\right|_{-l} \leq C h^{\gamma}(t-s)^{-(\gamma-l) / 2}\|g(s)\| \leq C h^{\gamma}(t-s)^{-1 / 2},
$$

and (1.8) follows using (1.4), which applies since now $\alpha \leq 1$ and hence $v \in \dot{H}^{\alpha}$, to bound $F_{h}(t) v$.

We next consider the case $0 \leq \gamma-\alpha-l \leq 2$ with $\gamma \geq l+1$. Recall that the error $e=u_{h}-u$ satisfies (1.9) with $\rho=-\left(P_{1}-I\right) u$ and $T_{h} e(0)=0$. We use Lemma 1.2 together with (1.3), now applicable since $\gamma-l \geq 1$, and (1.10) to obtain

$$
t|e(t)|_{-l, h} \leq C h^{\gamma} \sup _{s \leq t}\left\{s^{2}\left\|u_{t}(s)\right\|_{\gamma-l}+s\|u(s)\|_{\gamma-l}+\|\tilde{u}(s)\|_{\gamma-l}\right\} .
$$

By (1.6) and by (1.7), which applies since $\alpha \leq \gamma-l \leq 2+\alpha$,

$$
t|e(t)|_{-l, h} \leq C h^{\gamma} \sup _{s \leq t} s^{1-(\gamma-\alpha-l) / 2}=C h^{\gamma} t^{1-(\gamma-\alpha-l) / 2} .
$$

Similarly,

$$
t h^{l}\|e(t)\| \leq C h^{\gamma} t^{1-(\gamma-\alpha-l) / 2},
$$

and by (1.11) the desired result (1.8) follows in the present case, and thus generally for $0 \leq \gamma-\alpha-l \leq 2$. Note that this covers $r=2$, so that we may assume below that $r \geq 3$.

We shall now show by induction over $m \geq 2$ that (1.8) holds for $m-1<\gamma-\alpha-l \leq$ $m$. We have already seen this to be true for $m=2$, and assume it now to hold for 
some value $m$. To carry out the induction step, let $m<\gamma-\alpha-l \leq m+1$. Then, since $m-1<\gamma-\alpha-(l+1) \leq m$, we have by the induction assumption

$$
|e(s)|_{-(l+1), h} \leq C h^{\gamma} s^{-(\gamma-\alpha-(l+1)) / 2},
$$

provided that $l+1 \leq r-2$, which we shall now assume. This is automatically satisfied for $m \geq 3$ since $l+1<\gamma-m+1 \leq r-m+1$, but the case $m=2$ needs separate consideration. For $m \geq 3$, by Lemma 1.1 and (1.6) we obtain, for $k \geq(m+1) / 2$,

$$
\begin{aligned}
t^{k}|e(t)|_{-l, h} & \leq C \sup _{s \leq t}\left\{h^{\gamma} s^{k+1}\left\|u_{t}(s)\right\|_{\gamma-l}+h^{\gamma} s^{k}\|u(s)\|_{\gamma-l}+s^{k-1 / 2}|e(t)|_{-(l+1), h}\right\} \\
& \leq C h^{\gamma} \sup _{s \leq t} s^{k-(\gamma-\alpha-l) / 2} \leq C h^{\gamma} t^{k-(\gamma-\alpha-l) / 2}
\end{aligned}
$$

or

$$
|e(t)|_{-l, h} \leq C h^{\gamma} t^{-(\gamma-\alpha-l) / 2}
$$

Similarly,

$$
\|e(t)\| \leq C h^{\gamma-l} t^{-(\gamma-\alpha-l) / 2},
$$

so that the desired result follows by (1.11).

However, for the proof to be complete, we still need to show (1.8) for the case $2 \leq \gamma-\alpha-l \leq 3$, which has only been achieved so far if $l+1 \leq r-2$. From $l+1<\gamma-\alpha-1 \leq r-\alpha-1$ we see that this is satisfied if $\alpha \geq 1$. For $0 \leq \alpha<1$ we have, using $\gamma \leq 2+2 \alpha$, that $l+1<2+\alpha-1 \leq 2$ and hence $l+1 \leq r-2$ if $r \geq 4$. It remains to consider $\alpha<1$ together with $r=3$. But then it is enough to treat $0 \leq l<\alpha$ since $l \geq \alpha$ implies $\gamma-\alpha-l \leq 2+\alpha-l \leq 2$, which is covered by our first case. We shall see that (1.8) holds for $r=3, \alpha<1$ and $l$ replaced by $l^{\prime}=0$ and $\alpha$. For $l^{\prime}=0$ this follows by our previous argument since then $l^{\prime}+1=1=r-2$. For $l^{\prime}=\alpha<1$ we have $0 \leq \gamma-2 \alpha=\gamma-\alpha-l^{\prime} \leq 2$ since $\gamma>2$ and $\gamma \leq 2+2 \alpha$, so that (1.8) is valid by the first case. The desired result now follows for $0<l<\alpha$ by the obvious convexity inequality.

The proof of Theorem 1.1 is now complete.

We shall also need the following result.

THEOREM 1.2. Let $1+\alpha+l \leq r, 0 \leq l \leq r-2$. Then there exists a constant $C=C(R)$ such that, for the error in (1.2),

$$
\left\|u_{h}-u\right\|_{L_{2}\left(I ; \dot{H}^{-l}(\Omega)\right)} \leq C h^{1+\alpha+l} \quad \text { if }\|u\|_{L_{2}\left(I ; H^{1+\alpha}(\Omega)\right)} \leq R .
$$

Proof. From the error equation (1.9) we have after multiplication by $T_{h}^{l} e$ and integration, since $T_{h} e(0)=0$,

$$
\frac{1}{2}|e(t)|_{-l-1, h}^{2}+\int_{0}^{t}|e|_{-l, h}^{2} d s \leq \frac{1}{2} \int_{0}^{t}|\rho|_{-l, h}^{2} d s+\frac{1}{2} \int_{0}^{t}|e|_{-l, h}^{2} d s
$$

whence

$$
\int_{0}^{t}|e|_{-l, h}^{2} d s \leq \int_{0}^{t}|\rho|_{-l, h}^{2} d s
$$

Using (1.10) and the error estimates (1.3) for the elliptic projection we have

$$
|\rho(t)|_{-l, h} \leq C h^{1+\alpha+l}\|u(t)\|_{1+\alpha} \quad \text { for } t \in I .
$$

The desired result now follows by (1.11), completing the proof of Theorem 1.2. 
We shall next show that the assumptions of Theorems 1.1 and 1.2 are satisfied for the solution of the semilinear problem (0.1) with $v \in \mathscr{F}_{\alpha}$. Recall that for $\alpha \leq 2$, $\mathscr{F}_{\alpha}=\dot{H}^{\alpha} \cap L_{\infty}$, and $F_{\alpha}(v)$ equals the norm $\max \left(|v|_{\alpha},\|v\|_{L_{\infty}}\right)$. We begin with the assumptions of Theorem 1.1 and remark that the major part of the proof is already given in [2].

LEMMA 1.3. Let $0 \leq \alpha<2$ and let $u$ be the solution of (0.1). Then there exists a constant $C=C(R)$ such that (1.6) and (1.7) hold for all $v \in \mathscr{F}_{\alpha}$ with $F_{\alpha}(v) \leq R$.

Proof. In [2, Theorem 2.2] it was proved that if $0 \leq \beta-\alpha \leq 5,2 j \leq \beta$, then, with $C=C(R)$,

$$
\left\|D_{t}^{j} u(t)\right\|_{\beta-2 j} \leq C t^{-(\beta-\alpha) / 2} \text { for } F_{\alpha}(v) \leq R, t \in I .
$$

In particular, the inequality (1.6) is verified for $j=0$ and $\alpha \leq \mu \leq 5+\alpha$, which covers our case since $2+2 \alpha<5+\alpha$. For $j=1$, (1.6) only follows for $\mu \leq 3+\alpha$, and this shows the desired estimate only if $0 \leq \alpha \leq 1$. We shall now show

$$
\left\|u_{t}(t)\right\|_{4+\alpha} \leq C t^{-3} \text { for } 1 \leq \alpha<2, t \in I .
$$

Together with the estimate (1.6) for $\mu=3+\alpha$, this implies (1.6) with $j=1$ for $3+\alpha \leq \mu \leq 4+\alpha$ by interpolation. Since $2+2 \alpha$ is in this interval for $1 \leq \alpha<2$, the proof of (1.6) would then be complete.

We shall show (1.15) by successively demonstrating (1.14) with $\beta=6+\alpha$ for $j=3,2$, and 1 . For $j=3, w=u_{t t t}$ satisfies

$$
w_{t}-\Delta w=D_{t}^{3} f(u)=f^{\prime \prime \prime}(u) u_{t}^{3}+3 f^{\prime \prime}(u) u_{t} u_{t t}+f^{\prime}(u) u_{t t t} .
$$

By Duhamel's principle we have

$$
w(t)=E(t / 2) w(t / 2)+\int_{t / 2}^{t} E(t-s) D_{t}^{3} f(u(s)) d s=E(t / 2) w(t / 2)+I(t) .
$$

Here, by (1.5) and (1.14), which may be applied since $6-\alpha \leq 5$,

$$
\|E(t / 2) w(t / 2)\|_{\alpha} \leq C t^{-\alpha / 2}\left\|u_{t t t}(t / 2)\right\| \leq C t^{-\alpha / 2} t^{-(6-\alpha) / 2}=C t^{-3} .
$$

Further, using Sobolev's inequality,

$$
\begin{aligned}
\left\|D_{t}^{3} f(u)\right\| & \leq C\left(\left\|u_{t}\right\|_{L_{6}}^{3}+\left\|u_{t t}\right\| \cdot\left\|u_{t}\right\|_{L_{\infty}}+\left\|u_{t t t}\right\|\right) \\
& \leq C\left(\left\|u_{t}\right\|_{1}^{3}+\left\|u_{t t}\right\| \cdot\left\|u_{t}\right\|_{2}+\left\|u_{t t t}\right\|\right)
\end{aligned}
$$

and hence, using known cases of (1.14),

$$
\begin{aligned}
\|I(t)\|_{\alpha} & \leq C \int_{t / 2}^{t}(t-s)^{-\alpha / 2}\left\|D_{t}^{3} f(u(s))\right\| d s \\
& \leq C t^{1-\alpha / 2}\left(t^{-3(3-\alpha) / 2}+t^{-(4-\alpha) / 2} t^{-(4-\alpha) / 2}+t^{-(6-\alpha) / 2}\right) \leq C t^{-3},
\end{aligned}
$$

which shows the case $\beta=6+\alpha, j=3$ of (1.14).

We next take $j=2$. Since $\Delta u_{t t}=u_{t t t}-D_{t}^{2} f(u)$, we have by elliptic regularity

$$
\left\|u_{t t}\right\|_{2+\alpha} \leq C\left\|u_{t t t}\right\|_{\alpha}+C\left\|D_{t}^{2} f(u)\right\|_{\alpha},
$$

and, by the case just considered, it suffices to bound the second term. We now use the inequality

$$
\|\varphi \psi\|_{\alpha} \leq\|\varphi\|_{2}\|\psi\|_{\alpha} \text { for } 1 \leq \alpha \leq 2
$$


which follows by interpolation from the cases $\alpha=1$ and 2. We also apply Moser's lemma (cf. [3]): For any positive integer $k$,

$$
\|f(u)\|_{k} \leq C(K)\left(1+\|u\|_{k}\right) \text { for }\|u\|_{L_{\infty}} \leq K .
$$

We obtain

$$
\begin{aligned}
\left\|D_{t}^{2} f(u)\right\|_{\alpha} & =\left\|f^{\prime \prime}(u) u_{t}^{2}+f^{\prime}(u) u_{t t}\right\|_{\alpha} \leq\left\|f^{\prime \prime}(u)\right\|_{2}\left\|u_{t}^{2}\right\|_{\alpha}+\left\|f^{\prime}(u)\right\|_{2}\left\|u_{t t}\right\|_{\alpha} \\
& \leq C\left(1+\|u\|_{2}\right)\left(\left\|u_{t}\right\|_{2}\left\|u_{t}\right\|_{\alpha}+\left\|u_{t t}\right\|_{\alpha}\right)
\end{aligned}
$$

so that, by known cases of (1.14),

$$
\left\|D_{t}^{2} f(u)\right\|_{\alpha} \leq C t^{-(2-\alpha) / 2}\left(t^{-(4-\alpha) / 2} t^{-1}+t^{-2}\right) \leq C t^{-3}
$$

and the case $\beta=6+\alpha, j=2$ of (1.14) follows.

For the final step of the proof of (1.15) we use again elliptic regularity to obtain

$$
\left\|u_{t}\right\|_{4+\alpha} \leq C\left\|\Delta u_{t}\right\|_{2+\alpha} \leq C\left(\left\|u_{t t}\right\|_{2+\alpha}+\left\|D_{t} f(u)\right\|_{2+\alpha}\right) .
$$

Again, it only remains to consider the last term. Here we shall use

$$
\|\varphi \psi\|_{\beta} \leq\|\varphi\|_{3}^{4-\beta}\|\varphi\|_{4}^{\beta-3}\|\psi\|_{\beta} \quad \text { for } 3 \leq \beta \leq 4
$$

which follows by interpolation from the cases $\beta=3$ and $\beta=4$. Together with Moser's lemma, this gives

$$
\left\|D_{t} f(u)\right\|_{2+\alpha}=\left\|f^{\prime}(u) u_{t}\right\|_{2+\alpha} \leq C\left(1+\|u\|_{3}\right)^{2-\alpha}\left(1+\|u\|_{4}\right)^{\alpha-1}\left\|u_{t}\right\|_{2+\alpha}
$$

whence

$$
\left\|D_{t} f(u(t))\right\|_{2+\alpha} \leq C t^{-(2-\alpha)(3-\alpha) / 2} t^{-(\alpha-1)(4-\alpha) / 2} t^{-2}=C t^{-3} .
$$

We have now proved (1.15).

It remains to prove (1.7). For this purpose we show this inequality for $\mu=\alpha$ and $\mu=2+\alpha$, from which it then follows in general by interpolation. Since $\|u(t)\|_{\alpha}$ is bounded on $I$, we have

$$
\|\tilde{u}(t)\|_{\alpha} \leq \int_{0}^{t}\|u(s)\|_{\alpha} d s \leq C t \quad \text { for } t \in I .
$$

As concerns $\mu=2+\alpha$, we note that by integration of the differential equation we have

$$
u(t)-v-\Delta \tilde{u}(t)=\tilde{f}(u(t)) \text { for } t \in I,
$$

and hence by elliptic regularity,

$$
\|\tilde{u}\|_{2+\alpha} \leq C\|\Delta \tilde{u}\|_{\alpha} \leq C\left\{\|v\|_{\alpha}+\|u\|_{\alpha}+\|\tilde{f}(u)\|_{\alpha}\right\} \leq C\left\{1+\|f(u)\|_{\alpha}\right\} .
$$

For the last term on the right we note that with $k=[\alpha]+1$,

$$
\begin{aligned}
\|f(u(s))\|_{\alpha} & \leq C\|f(u(s))\|^{1-\alpha / k}\|f(u(s))\|_{k}^{\alpha / k} \\
& \leq C\left(1+\|u(s)\|_{k}\right)^{\alpha / k} \leq C s^{-(2-\alpha) \alpha / 4} \quad \text { for } t \in I,
\end{aligned}
$$

so that

$$
\|\tilde{f}(u)(t)\|_{\alpha} \leq C \int_{0}^{t} s^{-(2-\alpha) \alpha / 4} d s \leq C \int_{0}^{t} s^{-1 / 4} d s \leq C .
$$

By (1.16) this proves (1.7) for $\mu=2+\alpha$, which completes the proof of Lemma 1.3.

We finally turn to the assumptions of Theorem 1.2. 
LEMMA 1.4. Let $0 \leq \alpha \leq 2$. Then there exists a constant $C=C(R)$ such that $v \in \mathscr{F}_{\alpha}$ implies that the solution $u$ of $(0.1)$ belongs to $L_{2}\left(H^{1+\alpha}\right)$ and

$$
\|u\|_{L_{2}\left(H^{1+\alpha}\right)} \leq C \quad \text { for } F_{\alpha}(v) \leq R .
$$

Proof. Consider first the case $0 \leq \alpha \leq 1$ and multiply the differential equation in (0.1) by $(-\Delta)^{\alpha} u$ in $L_{2}$, which is legitimate since $u(t) \in \dot{H}^{2}$ for $t \in I$. We obtain

$$
\frac{1}{2} \frac{d}{d t}|u|_{\alpha}^{2}+|u|_{1+\alpha}^{2}=\left(T^{(1-\alpha) / 2} f(u),(-\Delta)^{(1+\alpha) / 2} u\right) \leq \frac{1}{2}|f(u)|_{\alpha-1}^{2}+\frac{1}{2}|u|_{1+\alpha}^{2},
$$

whence by integration

$$
\int_{0}^{t}|u|_{1+\alpha}^{2} d s \leq|v|_{\alpha}^{2}+\int_{0}^{t}\|f(u)\|^{2} d s \leq C .
$$

For $1 \leq \alpha \leq 2$, we have by elliptic regularity

$$
\begin{aligned}
\int_{0}^{t}\|u\|_{1+\alpha}^{2} d s & \leq C \int_{0}^{t}\|\Delta u\|_{\alpha-1}^{2} d s \\
& \leq C \int_{0}^{t}\left\|u_{t}\right\|_{\alpha-1}^{2} d s+C \int_{0}^{t}\|f(u)\|_{\alpha-1}^{2} d s
\end{aligned}
$$

Here,

$$
\int_{0}^{t}\|f(u)\|_{\alpha-1}^{2} d s \leq \int_{0}^{t}\|f(u)\|_{1}^{2} d s \leq C \int_{0}^{t}\left(1+|u|_{1}^{2}\right) d s \leq C \quad \text { for } t \in I .
$$

To estimate the first term on the right in (1.17), multiply the equation (0.1) by $(-\Delta)^{\alpha-1} u_{t}$ to obtain

$$
\left|u_{t}\right|_{\alpha-1}^{2}+\frac{1}{2} \frac{d}{d t}|u|_{\alpha}^{2} \leq\left(f(u),(-\Delta)^{\alpha-1} u_{t}\right)
$$

which after integration becomes

$$
\begin{aligned}
& \int_{0}^{t}\left|u_{t}\right|_{\alpha-1}^{2} d s+\frac{1}{2}|u(t)|_{\alpha}^{2} \\
& \quad \leq|v|_{\alpha}^{2}+\left.\left(f(u),(-\Delta)^{\alpha-1} u\right)\right|_{0} ^{t}-\int_{0}^{t}\left(f^{\prime}(u) u_{s},(-\Delta)^{\alpha-1} u\right) d s \\
& \quad=|v|_{\alpha}^{2}+\left.\left(T^{1-\alpha / 2} f(u),(-\Delta)^{\alpha / 2} u\right)\right|_{0} ^{t}-\int_{0}^{t}\left(T^{1-\alpha / 2}\left(f^{\prime}(u) u_{s}\right),(-\Delta)^{\alpha / 2} u\right) d s \\
& \quad \leq|v|_{\alpha}^{2}+C\left(\|f(u(t))\| \cdot|u(t)|_{\alpha}+\|f(u(0))\| \cdot|v|_{\alpha}+\int_{0}^{t}\left\|u_{s}\right\| \cdot|u|_{\alpha} d s\right) \\
& \quad \leq C+C \int_{0}^{t}\left\|u_{s}\right\| d s \leq C+C \int_{0}^{t} s^{-(1-\alpha / 2)} d s \leq C .
\end{aligned}
$$

This completes the proof of Lemma 1.4.

2. The Main $L_{2}$ Error Estimates. The main result in this section is the following

THEOREM 2.1. Let $0 \leq \alpha<2,2+2 \alpha \leq r$, and let $u_{h}$ and $u$ be the solutions of (0.2) and (0.1), respectively, with $v \in \mathscr{F}_{\alpha}$. Then there exist constants $C=C(R, \alpha)$ and $\nu=\nu(\alpha)<1$ such that

(2.1) $\left\|u_{h}(t)-u(t)\right\| \leq C h^{2+2 \alpha}\left(t^{-(2+\alpha) / 2}+\log (1 / h) t^{-\nu}\right) \quad$ for $F_{\alpha}(v) \leq R, t \in I$. 
By Duhamel's principle the exact and approximate solutions of $(0.1)$ satisfy the nonlinear integral equations

$$
u(t)=E(t) v+\int_{0}^{t} E(t-s) f(u(s)) d s
$$

and

$$
u_{h}(t)=E_{h}(t) P_{0} v+\int_{0}^{t} E_{h}(t-s) P_{0} f\left(u_{h}(s)\right) d s
$$

respectively. The proof of the theorem will depend on introducing as an auxiliary function $\hat{u}: I \rightarrow S_{h}$, the Galerkin solution of the linear nonhomogeneous equation with right-hand side $f(u(x, t))$, i.e.,

$$
\hat{u}_{h}(t)=E_{h}(t) P_{0} v+\int_{0}^{t} E_{h}(t-s) P_{0} f(u(s)) d s .
$$

Using (2.2), (2.3), and (2.4), we may then write for the error $e=u_{h}-u$, with $\hat{e}=\hat{u}_{h}-u$,

$$
e(t)=\hat{e}(t)+\int_{0}^{t} E_{h}(t-s) P_{0}\left(f\left(u_{h}(s)\right)-f(u(s))\right) d s .
$$

By the results of Section 1 we have access to a variety of estimates for $\hat{e}$ which will be applied in the analysis of (2.5). For this purpose we introduce an integral operator $J$ defined for functions $w=w(x, t)$ by

$$
(J w)(t)=\int_{0}^{t} E(t-s) f^{\prime}(u(s)) w(s) d s
$$

and rewrite (2.5) in the form

$$
e=g_{0}+g_{1}+g_{2}+J e
$$

where $g_{0}=\hat{e}$,

$$
g_{1}(t)=\int_{0}^{t}\left(E_{h}(t-s) P_{0}-E(t-s)\right)\left(f\left(u_{h}(s)\right)-f(u(s))\right) d s
$$

and

$$
g_{2}(t)=\int_{0}^{t} E(t-s)\left[\left(f\left(u_{h}(s)\right)-f(u(s))-f^{\prime}(u(s))\right)\left(u_{h}(s)-u(s)\right)\right] d s .
$$

Define now $e_{i}$ to be the solutions of the integral equations

$$
e_{i}=g_{i}+J e_{i}, \quad i=0,1,2 .
$$

By standard techniques for Volterra integral equations these equations have unique solutions, and it is clear from (2.6) that the error $e$ may be represented as $e=$ $e_{0}+e_{1}+e_{2}$. In estimating the different terms of the error, the following easily proved version of Gronwall's lemma will be used repeatedly.

LEMMA 2.1. If

$$
0 \leq \varphi(t) \leq \Gamma(t)+C \int_{0}^{t} \varphi(s) d s \quad \text { for } t \in I
$$

then

$$
\|\varphi\|_{L_{2}(I)} \leq C\|\Gamma\|_{L_{2}(I)}
$$


and

$$
0 \leq \varphi(t) \leq \Gamma(t)+C \int_{0}^{t} \Gamma(s) d s \quad \text { for } t \in I .
$$

For the proof of Theorem 2.1 we start by deriving some preliminary low-order estimates for $e$. By (2.5) we have

$$
\|e(t)\| \leq\|\hat{e}(t)\|+C \int_{0}^{t}\|e(s)\| d s
$$

so that by Lemma 2.1, Theorem 1.2 and Lemma 1.4 ,

$$
\|e(t)\|_{L_{2}\left(L_{2}\right)} \leq C\|\hat{e}(t)\|_{L_{2}\left(L_{2}\right)} \leq C h^{1+\alpha}
$$

and, using also Theorem 1.1,

(2.9) $\|e(t)\| \leq C h^{\sigma+\alpha}\left(t^{-\sigma / 2}+C \int_{0}^{t} s^{-\sigma / 2} d s\right) \leq C h^{\sigma+\alpha} t^{-\sigma / 2}$ for $\sigma<2, t \in I$.

We proceed to derive the more precise estimates needed for $e_{1}$ and $e_{2}$. By Lemma 2.1 ,

$$
\left\|e_{i}(t)\right\| \leq\left\|g_{i}(t)\right\|+C \int_{0}^{t}\left\|g_{i}(s)\right\| d s \quad \text { for } i=1,2 .
$$

Here, by the results (1.4) for the homogeneous problem and (2.9) with $\sigma=(2+\alpha) / 2$,

$$
\begin{aligned}
\left\|g_{1}(t)\right\| & \leq C \int_{0}^{t} C h^{(2+\alpha) / 2}(t-s)^{-(2+\alpha) / 4}\|e(s)\| d s \\
& \leq \int_{0}^{t} C h^{(2+\alpha) / 2}(t-s)^{-(2+\alpha) / 4} h^{(2+3 \alpha) / 2} s^{-(2+\alpha) / 4} d s \leq C h^{2+2 \alpha} t^{-\alpha / 2} .
\end{aligned}
$$

Hence, by (2.10),

$$
\left\|e_{1}(t)\right\| \leq C h^{2+2 \alpha} t^{-\alpha / 2}
$$

By Taylor's theorem we have, for some $\bar{u}(x, s)=\bar{u} \in \operatorname{int}\left[u_{h}, u\right]$,

$$
g_{2}(t)=\frac{1}{2} \int_{0}^{t} E(t-s) f^{\prime \prime}(\bar{u}) e(s)^{2} d s
$$

so that

$$
\left\|g_{2}(t)\right\| \leq C \int_{0}^{t}\|E(t-s)\|_{L_{1} \rightarrow L_{2}}\|e(s)\|^{2} d s .
$$

Since by Sobolev's lemma and (1.5), for $d / 4<\delta<1$,

$$
\|E(s) \psi\|_{L_{\infty}} \leq C\|E(s) \psi\|_{2 \delta} \leq C s^{-\delta}\|\psi\| \quad \text { for } s \in I,
$$

we find by duality, since $E(s)$ is selfadjoint on $L_{2}$, that

$$
\|E(t-s)\|_{L_{1} \rightarrow L_{2}} \leq C(t-s)^{-\delta} .
$$

Hence, by (2.12), (2.8) and (2.9) with $\sigma=1$,

$$
\begin{aligned}
\left\|g_{2}(t)\right\| & \leq C\left(\int_{0}^{t / 2}+\int_{t / 2}^{t}\right)(t-s)^{-\delta}\|e(s)\|^{2} d s \\
& \leq C t^{-\delta}\|e(s)\|_{L_{2}\left(L_{2}\right)}^{2}+C \int_{t / 2}^{t}(t-s)^{-\delta} h^{2+2 \alpha} s^{-1} d s \leq C h^{2+2 \alpha} t^{-\delta}
\end{aligned}
$$


and thus, by $(2.10)$, since $\delta<1$,

$$
\left\|e_{2}(t)\right\| \leq C h^{2+2 \alpha} t^{-\delta} \text {. }
$$

We finally estimate $e_{0}$. From (2.7) we see that $\hat{w}=e_{0}-\hat{e}$ satisfies the integral equation $\hat{w}=J \hat{e}+J \hat{w}$, and hence, by Lemma 2.1 and the triangle inequality,

$$
\left\|e_{0}(t)\right\| \leq\|\hat{e}(t)\|+\|J \hat{e}(t)\|+\int_{0}^{t}\|J \hat{e}(s)\| d s .
$$

We shall show that, with $\nu=\nu(\alpha)<1$ for $0 \leq \alpha<2$,

$$
\|J \hat{e}(t)\| \leq C h^{2+2 \alpha} t^{-\nu} \log (1 / h) .
$$

Together with Theorem 1.1, (2.14) implies that $\left\|e_{0}\right\|$ is bounded by the right-hand side of (2.1). In view of our above estimates (2.11) and (2.13) for $e_{1}$ and $e_{2}$, this would complete the proof of Theorem 2.1 .

For the purpose of showing (2.15) we first note that it suffices to consider the case $h^{2+2 \alpha} \leq t / 2$, since in the opposite case, $\|J \hat{e}(t)\|$ is trivially bounded by $C h^{2+2 \alpha}$. We now write $J \hat{e}(t)=J_{0}(t)+J_{1}(t)+J_{2}(t)$ corresponding to the intervals of integration $\left(0, h^{2+2 \alpha}\right),\left(h^{2+2 \alpha}, t / 2\right)$, and $(t / 2, t)$, and $\left\|J_{0}(t)\right\|$ is again trivially bounded by $C h^{2+2 \alpha}$. Further, by Theorem 1.1,

$$
\left\|J_{2}(t)\right\| \leq C \int_{t / 2}^{t}\|\hat{e}(s)\| d s \leq C h^{2+2 \alpha} \int_{t / 2}^{t} s^{-(2+\alpha) / 2} d s=C h^{2+2 \alpha} t^{-\alpha / 2} .
$$

It remains to estimate $J_{1}(t)$. We have

$$
\left\|J_{1}(t)\right\|=\sup _{\|w\|=1} \int_{h^{2+2 \alpha}}^{t / 2} Q(t, s ; w) d s
$$

where, with $\|w\|=1, \varphi=\varphi(s)=f^{\prime}(u(s))$ and $\psi=\psi(t-s)=E(t-s) w$,

$$
Q(t, s)=Q(t, s ; w) \equiv\left(E(t-s) f^{\prime}(u(s)) \hat{e}(s), w\right)=(\hat{e}, \varphi \psi) .
$$

We consider first $r \geq 4$, in which case an error estimate for $\hat{e}$ in $\dot{H}^{-2}$ is available, so that, since $\varphi \psi \in \dot{H}^{2}$,

$$
|Q| \leq C|\hat{e}|_{-2}\|\varphi \psi\|_{2} \leq C|\hat{e}|_{-2}\|\varphi\|_{2}\|\psi\|_{L_{\infty}}+C|\hat{e}|_{-2}\|\varphi\|_{L_{\infty}}\|\psi\|_{2}
$$

where the second inequality follows from the Gagliardo-Nirenberg inequality, as in the proof of Moser's lemma. We have $\|\varphi\|_{L_{\infty}} \leq C$, and from Moser's lemma and (1.6),

$$
\|\varphi(s)\|_{2} \leq C\left(1+\|u(s)\|_{2}\right) \leq C s^{-(2-\alpha) / 2} .
$$

Using (1.8) and Sobolev's lemma, we get, with $3 / 4<\delta<1$,

$$
\begin{aligned}
|Q(t, s)| & \leq C h^{2+2 \alpha} s^{-\alpha / 2}\left(s^{-(2-\alpha) / 2}(t-s)^{-\delta}+(t-s)^{-1}\right) \\
& \leq C h^{2+2 \alpha}\left(s^{-1} t^{-\delta}+s^{-\alpha / 2} t^{-1}\right) \quad \text { for } s \leq t / 2 .
\end{aligned}
$$

Hence, with $\nu=\max (\delta, \alpha / 2)$,

$$
\left\|J_{1}(t)\right\| \leq C h^{2+2 \alpha} \int_{h^{2+2 \alpha}}^{t / 2}\left(s^{-1} t^{-\delta}+s^{-\alpha / 2} t^{-1}\right) d s \leq C h^{2+2 \alpha} \log (1 / h) t^{-\nu} .
$$

This proves (2.15) and thus the theorem in case $r \geq 4$. For $r=3$, the estimate (2.16) is changed into

$$
|Q(t, s)| \leq C|\hat{e}|_{-1}\|\varphi\|_{1}\|\psi\|_{L_{\infty}}+C|\hat{e}|_{-1}\|\varphi\|_{L_{\infty}}\|\psi\|_{1} .
$$


The argument then proceeds as before with the obvious changes to show that

$$
\left|J_{1}(t)\right| \leq C h^{2+2 \alpha} \log (1 / h) t^{-\delta} .
$$

This completes the proof of Theorem 2.1.

In some cases it is possible to remove the logarithmic term in the estimate (2.1). We shall indicate how to do this for $0 \leq \alpha \leq 1, r \geq 4$, and also for $\alpha=0, r \geq 3$. It is clear from the above analysis that it suffices to consider the term $J_{1}(t)$.

We first note that for $u(s) \in H^{\beta} \cap L_{\infty}$ with $0 \leq \beta \leq 2$, it is possible to find $u_{2}(s)$, so that for $\tau>0$ given,

$$
\begin{aligned}
& \left\|u(s)-u_{2}(s)\right\| \leq C \tau^{\beta}\|u(s)\|_{\beta}, \\
& \left\|u_{2}(s)\right\|_{2} \leq C \tau^{-(2-\beta)}\|u(s)\|_{\beta},
\end{aligned}
$$

and

$$
\left\|u_{2}(s)\right\|_{L_{\infty}} \leq C\|u(s)\|_{L_{\infty}}
$$

where the constants do not depend on $\tau$. The function $u_{2}$ may be produced by extending $u$ smoothly to $R^{d}$ and then applying a suitable convolution operator, or, since only small $\tau$ are at issue $\left(u_{2}=0\right.$ works for large $\tau$ ), by taking $u_{2}$ as the $L_{2}$-projection into a suitable (fictitious) finite element space on $\Omega$. Applying this to the solution $u$ of (1.2), we set $\varphi_{2}=f^{\prime}\left(u_{2}\right)$ and $\beta=1+\alpha \leq 2$ to obtain

$$
\left\|\varphi-\varphi_{2}\right\|=\left\|f^{\prime}(u)-f^{\prime}\left(u_{2}\right)\right\| \leq C\left\|u-u_{2}\right\| \leq C \tau^{\beta}\|u\|_{\beta} .
$$

Further, by Moser's lemma, using (2.18),

$$
\left\|\varphi_{2}\right\|_{2} \leq C\left(1+\left\|u_{2}\right\|_{2}\right) \leq C\left(1+\tau^{-(2-\beta)}\|u\|_{\beta}\right) .
$$

Now, with notation as in the proof of Theorem 2.1,

$$
Q(t, s)=\left(\hat{e},\left(\varphi-\varphi_{2}\right) \psi\right)+\left(\hat{e}, \varphi_{2} \psi\right)
$$

and thus, for $s \leq t / 2$,

$$
\begin{aligned}
|Q(t, s)| & \leq\|\hat{e}\|\left\|\left(\varphi-\varphi_{2}\right) \psi\right\|+|\hat{e}|_{-2}\left\|\varphi_{2} \psi\right\|_{2} \\
& \leq C\|\hat{e}(s)\|\left\|\left(\varphi-\varphi_{2}\right)(s)\right\| t^{-\delta}+C|\hat{e}(s)|_{-2}\left(\left\|\varphi_{2}\right\|_{2} t^{-\delta}+\left\|\varphi_{2}\right\|_{L_{\infty}} t^{-1}\right) .
\end{aligned}
$$

Hence, integrating in time and using Cauchy-Schwarz's inequality,

$$
\begin{aligned}
\left\|J_{1}(t)\right\| \leq & C\|\hat{e}\|_{L_{2}\left(L_{2}\right)} \tau^{\beta}\|u\|_{L_{2}\left(H^{\beta}\right)} t^{-\delta} \\
& +C\|\hat{e}\|_{L_{2}\left(\dot{H}^{-2}\right)} \tau^{-(2-\beta)}\|u\|_{L_{2}\left(H^{\beta}\right)} t^{-\delta}+C\|\hat{e}\|_{L_{1}\left(\dot{H}^{-2}\right)} t^{-1} .
\end{aligned}
$$

From Lemma 1.4, $\|u\|_{L_{2}\left(H^{\beta}\right)}$ is bounded $(\beta=1+\alpha)$. Using Theorem 1.2 and (1.8), we obtain

$$
\left\|J_{1}(t)\right\| \leq C\left(h^{\beta} \tau^{\beta} t^{-\delta}+h^{2+\beta} \tau^{-(2-\beta)} t^{-\delta}+h^{2+2 \alpha} t^{-\alpha / 2}\right),
$$

and taking $\tau=h$,

$$
\left\|J_{1}(t)\right\| \leq C h^{2+2 \alpha}\left(t^{-\delta}+t^{-\alpha / 2}\right)
$$

which together with our previous estimates yields

$$
\|e(t)\| \leq C h^{2+2 \alpha} \tau^{-(2+\alpha) / 2} \quad \text { for } t \in I .
$$

In the case $\alpha=0, r=3$, we use (2.7), Moser's lemma and Theorem 1.1 to obtain

$$
|Q(t, s)| \leq C|\hat{e}(s)|_{-1}\left(1+\|u(s)\|_{1}\right) t^{-\delta}+C h^{2} s^{-1 / 2} t^{-1 / 2} \quad \text { for } s \leq t / 2 .
$$

Proceeding as before, using Theorem 1.2, this gives $\left\|J_{1}(t)\right\| \leq C h^{2} t^{-\delta}$, so that finally $\|e(t)\| \leq C h^{2} t^{-1}$ for $t \in I$. 
3. Error Estimates for Gradients and in Maximum Norm. This section will briefly indicate how Theorem 2.1 can be used to derive the estimates mentioned in the title. The techniques are those of [2, Sections 4 and 5], and we shall merely point out the slight modifications needed. We assume that the reader is familiar with the notation of those two sections. For our first result we assume in addition to (1.3) that, with $\Omega_{h} \subseteq \Omega$ denoting the mesh domains, we have for $1 \leq s \leq r$,

$$
\left\|\nabla P_{1} w-\nabla w\right\|_{L_{2}\left(\Omega_{h}\right)} \leq C h^{s-1}\|w\|_{s} \text { for } w \in H^{s} \cap H_{0}^{1} .
$$

THEOREM 3.1. Let $0 \leq \alpha<2,2+2 \alpha \leq r-1$. Then there exists a constant $C=C(R, \alpha)$ such that for the error in $(0.2)$

$$
\left\|\nabla u_{h}(t)-\nabla u(t)\right\|_{L_{2}\left(\Omega_{h}\right)} \leq C h^{2+2 \alpha} \log (1 / h) t^{-3 / 2-\alpha / 2} \quad \text { for } F_{\alpha}(v) \leq R, t \in I .
$$

Sketch of Proof. By (3.1) and (1.6) it clearly suffices to estimate $\theta=u_{h}-P_{1} u$. Proceeding as in [2, Theorem 4.1], but multiplying the basic equation for $\theta$ by $t^{5}$ rather than $t^{3}$, we find with $e=u_{h}-u$,

$$
t^{5}\|\nabla \theta(t)\|^{2} \leq C \int_{0}^{t}\left(s^{3}\|e\|^{2}+s^{3}\|\rho\|^{2}+s^{5}\left\|\rho_{t}\right\|^{2}\right) d s .
$$

Now use the bound of Theorem 2.1 for $e$ and (1.6) for $u$ and $u_{t}$ to obtain

$$
t^{5}\|\nabla \theta(t)\|^{2} \leq C h^{2(2+2 \alpha)} \log ^{2}(1 / h) t^{2-\alpha},
$$

which proves Theorem 3.1.

As for maximum norm error estimates, we have the following result, assuming that, with $\delta=0$ or 1 ,

$$
\left\|P_{1} w-w\right\|_{L_{\infty}} \leq C h^{s}(\log (1 / h))^{\delta}\|w\|_{W_{\infty}^{s}} \text { for } 0 \leq s \leq r, w=0 \text { on } \partial \Omega .
$$

THEOREM 3.2. Let $0 \leq \alpha<2,2+2 \alpha \leq r$. For any $t_{0} \in I$ there exists $C=C\left(R, t_{0}, \alpha\right)$ such that for the error in $(0.2)$

$$
\left\|u_{h}(t)-u(t)\right\|_{L_{\infty}} \leq C h^{2+2 \alpha} \log (1 / h) \quad \text { for } F_{\alpha}(v) \leq R, t_{0} \leq t \leq t^{*} .
$$

Sketch of Proof. For $\rho$ we have

$$
\|\rho(t)\|_{L_{\infty}} \leq C h^{2+2 \alpha}(\log (1 / h))^{\delta}\|u\|_{W_{\infty}^{2+2 \alpha}} \leq C(t) h^{2+2 \alpha}(\log (1 / h))^{\delta} .
$$

For $\theta$, following [2, Proof of Theorem 5.1], with $\varepsilon$ small positive,

$$
\left.t^{\beta}\|\theta(t)\|_{L_{\infty}} \leq C \int_{0}^{t}(t-s)^{-d / 4-\varepsilon}\left[s^{\beta-1}(\|e\|+\|\rho\|)+s^{\beta}\left\|\rho_{t}\right\|\right)\right] d s,
$$

whereupon Theorem 2.1 and (1.6) give, for $\beta$ large enough,

$$
t^{\beta}\|\theta(t)\|_{L_{\infty}} \leq C h^{2+2 \alpha} \log (1 / h) t^{\beta-\alpha / 2-d / 4-\varepsilon},
$$

and Theorem 3.2 follows.

To estimate the exact time-dependence in (3.2), one would, perhaps, use Sobolev's inequality, so that

$$
\|u\|_{W_{\infty}^{2+2 \alpha}} \leq C\|u\|_{2+2 \alpha+d / 2+\varepsilon} .
$$

Since the gap $(2+2 \alpha+d / 2+\varepsilon)-\alpha$ may now exceed 5 , a further extension of $[2$, Theorem 2.2] is needed. For the sake of brevity we shall not pursue the matter further. 
Finally, for the maximum norm of the error in the gradient we have the following result, assuming that, with $\delta=0$ or 1 ,

$\left\|\nabla P_{1} w-\nabla w\right\|_{L_{\infty}\left(\Omega_{h}\right)} \leq C h^{s-1}(\log (1 / h))^{\delta}\|w\|_{W_{\infty}^{s}} \quad$ for $1 \leq s \leq r, w=0$ on $\partial \Omega$.

THEOREM 3.3. Let $0 \leq \alpha<2,2+2 \alpha \leq r-1$. For any $t_{0} \in I$ there exists $C=C\left(R, t_{0}, \alpha\right)$ such that for the error in $(0.2)$

$$
\left\|\nabla u_{h}(t)-\nabla u(t)\right\|_{L_{\infty}\left(\Omega_{h}\right)} \leq C h^{2+2 \alpha} \log (1 / h) \quad \text { for } F_{\alpha}(v) \leq R, t_{0} \leq t \leq t^{*} .
$$

Sketch of Proof. The estimate for $\nabla \rho$ is treated as in Theorem 3.2. We next note the following easily proved extension of [2, Lemma 5.2]:

$$
\left\|\nabla E_{h}(t-s) w_{h}\right\|_{L_{\infty}} \leq C(t-s)^{-d / 4-i-\varepsilon}\left\|w_{h}\right\|_{1-i}, \quad i=0,1 .
$$

Recalling from [2, Proof of Theorem 5.1] that

$$
t^{\beta} \theta(t)=\left(\int_{0}^{t / 2}+\int_{t / 2}^{t}\right) E_{h}(t-s)\left[s^{\beta} P_{0}\left(\omega e-\rho_{t}\right)+\beta s^{\beta-1} \theta\right] d s \equiv I_{1}+I_{2},
$$

we proceed to estimate $I_{1}$ and $I_{2}$. For $I_{1}$ we use (3.3) with $i=1$ to obtain

$$
t^{\beta}\left\|\nabla I_{1}(t)\right\|_{L_{\infty}} \leq C \int_{0}^{t / 2}(t-s)^{-d / 4-1-\varepsilon}\left[s^{\beta}\left(\|\omega e\|+\left\|\rho_{t}\right\|\right)+s^{\beta-1}(\|e\|+\|\rho\|)\right] d s,
$$

whereupon using Theorem 2.1 and (1.6),

$$
\left\|\nabla I_{1}(t)\right\|_{L_{\infty}} \leq C h^{2+2 \alpha} \log (1 / h) .
$$

For $I_{2}$ we apply (3.3) with $i=0$ and find

$$
\begin{array}{r}
t^{\beta}\left\|\nabla I_{2}(t)\right\|_{L_{\infty}} \leq C \int_{t / 2}^{t}(t-s)^{-d / 4-\varepsilon}\left[s^{\beta}\left(\|\omega e\|_{H^{1}\left(\Omega_{h}\right)}+\left\|\rho_{t}\right\|_{H^{1}\left(\Omega_{h}\right)}\right)\right. \\
\left.+s^{\beta-1}\left(\|e\|_{H^{1}\left(\Omega_{h}\right)}+\|\rho\|_{H^{1}\left(\Omega_{h}\right)}\right)\right] d s
\end{array}
$$

so that use of Theorem 3.1 and the smoothness of $u$ on $I$ yields

$$
\left\|\nabla I_{2}(t)\right\|_{L_{\infty}} \leq C h^{2+2 \alpha} \log (1 / h) .
$$

This proves Theorem 3.3.

Acknowledgments. This work was started when the first author visited the Mathematical Sciences Institute, Cornell University, and was completed when the last two authors were visitors at the Centre for Mathematical Analysis, Australian National University. The third author also acknowledges support from the U.S. Army through the Mathematical Sciences Institute and from the National Science Foundation.

IRISA-Mathematiques

Université de Rennes I

Campus de Beaulieu

F-35042 Rennes Cedex, France

E-mail: crouzeix@irisa.irisa.fr

Department of Mathematics

Chalmers University of Technology

S-41296 Göteborg, Sweden

E-mail: cmsvt@seguc 21.bitnet 
Department of Mathematics

Cornell University

Ithaca, New York 14853

E-mail: wahlbin@mssun7.msi.cornell.edu

1. H.-P. HELFRICH "Error estimates for semidiscrete Galerkin type approximations to semilinear evolution equations with nonsmooth initial data," Numer. Math., v. 51, 1987, pp. 559-569.

2. C. JOHNSON, S. LARSSON, V. ThOMÉE \& L. B. WAHLBIN, "Error estimates for spatially discrete approximations of semilinear parabolic equations with nonsmooth initial data," Math. Comp., v. 49, 1987, pp. 331-357.

3. J. MOSER, "A rapidly convergent iteration method and nonlinear partial differential equations. I," Ann. Scuola Norm. Sup. Pisa, v. 20, 1966, pp. 265-315.

4. V. ThомéE, Galerkin Finite Element Methods for Parabolic Problems, Springer, New York, 1984. 